\title{
A Multi-Class Classification Based Approach for Remaining Useful Life (RUL) Prediction of Li-Ion Battery
}

\author{
Yuvraj Gupta ${ }^{1}$, Partho Ghosh², D.J. Dahigaonkar ${ }^{3}$ and P.A. Dwaramwar ${ }^{4}$ \\ ${ }^{1,2}$ Student, Department of Electronics and Communication Engineering, \\ Shri Ramdeobaba College of Engineering and Management, Nagpur, India \\ ${ }^{3}$ Associate Professor, Department of Electronics and Communication Engineering, \\ Shri Ramdeobaba College of Engineering and Management, Nagpur, India \\ ${ }^{4}$ Associate Professor, Department of Electronics Engineering, Shri Ramdeobaba \\ College of Engineering and Management, Nagpur, India
}

\section{ABSTRACT}

Estimation of Remaining Useful Life of the battery reduces the risk of battery failure and also helps in the optimization of the battery life by proposing its replacement at a proper time. This paper proposes a new RUL prediction technique based on the Multi-Class Classification approach. In order to predict the group membership of data instances, we have utilized the classification approach of the Machine learning technique. To simplify the issues related to classification, a neural network approach is deployed. To estimate the RUL, multiple measurable data features from the battery monitoring system are considered such as capacity, voltage, current, and temperature charging/discharging profiles. This research work utilizes the Li-Ion battery dataset of NASA Prognostics Center of Excellence Data Repository to verify the efficacy of the proposed machine learning model. The proposed method eliminates the need to rely on complicated battery electrochemical principles. This is very crucial as the technique can be deployed for various types of batteries. Accordingly, the RUL estimation tool proposed in this paper may benefit the upcoming automotive industry, particularly to the electric vehicles.

KEY WORDS: REMAINING USEFUL LIFE (RUL), BATTERY, MULTI-CLASS CLASSIFICATION, MULTI-LAYER NEURAL NETWORK.

\section{INTRODUCTION}

In today's world, battery is a daily necessity. Without battery the gadgets like mobile phones, laptops, remote controls etc. are no conceivable. We mechanically make use batteries in everyday life. The battery performance over long durations gets deteriorated due to many factors

\section{ARTICLE INFORMATION}

*Corresponding Author: guptays@rknec.edu

Received 19th Oct 2020 Accepted after revision 28th Dec 2020

Print ISSN: 0974-6455 Online ISSN: 2321-4007 CODEN: BBRCBA

Thomson Reuters ISI Web of Science Clarivate Analytics USA and Crossref Indexed Journal

\section{Clarivate
Analytics}

NAAS Journal Score 2020 (4.31)

A Society of Science and Nature Publication,

Bhopal India 2020. All rights reserved.

Online Contents Available at: http//www.bbrc.in/

Doi: $h t t p: / / d x$.doi.org/10.21786/bbrc/13.14/114 such as various chemical reactions, increase of service life, loss of battery material, etc. which may change the normal operation of electrical devices. Therefore, the battery states like Remaining Useful Life (RUL) of a battery and the State of Charge (SOC) of a battery has become a topic of research in engineering domain. Out of the many types of batteries available in the market, the Lithium-ion batteries are most preferred due to its convenient size \&t weight, longevity and high energy density. Since batteries are a significant component of many electrical systems, their failure can degrade the power system performance and may result in the system failure and increased costs. The RUL is a predictive technique that assures safe and stable condition of a battery to the users. An effective method to predict the RUL of a Lithium-ion battery is discussed in this paper. RUL estimations can be based 
on estimation of the properties of battery capacity and the state of charging and discharging, with the given failure threshold. Out of these, we have utilized capacity as battery health indicator for RUL predictions as the change in battery capacity directly indicates the battery health condition.

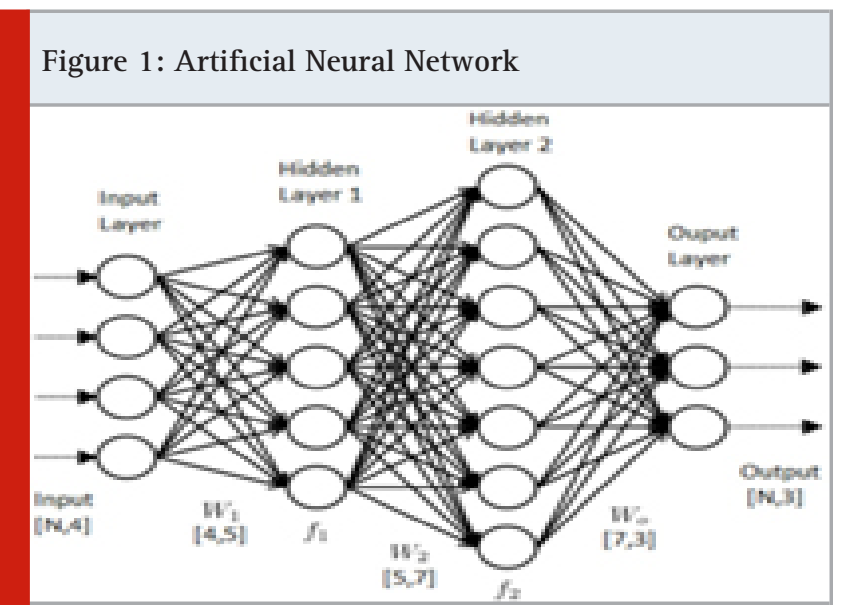

\section{Related Algorithms}

a. Multi-Layer Neural Network: Multi-Layer Neural Network comprises one or more concealed layers (excluding one input and one output layer). The advantage of multi-layer perceptron over single layer is multi-layer perceptron can be trained for non-linear functions too. The input to this neuron can be $\mathrm{x} 1, \mathrm{x} 2 \ldots$ $\mathrm{x} 3$ (and $\mathrm{a}+1$ bias term) with outputs $\mathrm{f}$ (summed inputs + bias), where $f($.$) is called as activation function. In$ addition to the customary inputs that the node receives, the main function of Bias is to furnish every node with a trainable constant value. Every activation function takes a solitary number and executes a distinctive fixed computation thereon.

Multi-Class Classification Using Deep Learning Model: Multiclass classification function contains more than two classes. In such classification, a sample can only have one class out of the multitude of accessible classes. The classification adopted in this work has many coaching examples which are separated into $\mathrm{K}$ separate classes, and based on this approach, a machine learning model was deployed to predict previously unseen data belongs to which of these classes. The training data helps to assimilate patterns unique to every class and use those patterns to forecast the membership of future data.

A neural network can be used as a classifier by just appending a softmax function onto the last layer. This function culminates a probability distribution over $\mathrm{K}$ classes and raises an output vector of length $\mathrm{K}$. The probability that the input belongs to the corresponding class is specified by each element of the vector. The probable class is then chosen by selecting the index of that vector having the foremost effective probability.

Multi-Class Classification And Softmax Regression: The method of logistic regression could be a binary classifier naturally. However, it may also be used for multi-class classifications with $\mathrm{K}>2$ classes. First, the strategy of One-Vs.-Rest (1VR) is accustomed to convert a K-class problem $(K>2$ ) into $K$ binary problems. Specifically, we regroup the $\mathrm{K}$ classes $\mathrm{C} 1, \ldots, \mathrm{CK}$ into two classes $\mathrm{C}(+)=$ $C_{i}$ and $C-=\left\{C_{j} \mid j=1, \ldots, K, j \neq i\right\}$, and get the corresponding discriminant (decision) function $\mathrm{f}(\mathrm{x})$ of the binary problem:

$$
\text { if } f_{i}(x)\left\{\begin{array} { l } 
{ > 0 } \\
{ < 0 }
\end{array} , \text { then } \left\{\begin{array}{lll}
x & \in C_{+} \\
x & \in & C_{-}
\end{array}\right.\right.
$$

which represents quantitatively how much a given $\mathrm{x}$ belongs to class $C_{+}=C_{i}$, instead of $C$ - containing all remaining $\mathrm{K}-1$ classes. This process is repeated $\mathrm{K}$ times for all $\mathrm{i}=1 \quad \mathrm{~K}$ to oet

$$
f_{k}(x)=\max \left\{f_{1}(x), \ldots, f_{K}(x)\right\},(k=1, \ldots, K)
$$

which indicates $x$ belongs to class $C_{k}$, instead of any of the remaining $\mathrm{K}-1$ classes.

As a substitute, One-Vs.-One (1V1) is another strategy to change a twofold classifier over to a multi-class classifier. In view of this method, an unlabeled $\mathrm{x}$ is assessed into a category that receives the utmost votes out of the (K (K-1)) /2 binary classifications between every pair of the $\mathrm{K}$ classes. Instead of converting the binary logistic regression, we will also generalize it into multinomial logistic regression, or softmax regression, for multi-class problems. In view of this case, the variable quantity y could be a categorical variable that takes anyone of $\mathrm{K}$ distinct values representing $\mathrm{K}>2$ different classes. While in logistic regression the logistic function $\sigma$ is utilized to show the likelihood of the function of $\mathrm{y}=1$ by a softmax work characterized as:

$$
p(y=i / x)=\emptyset_{i}\left(w_{i}^{T} x\right)=\frac{\exp \left(w_{i}^{T} x\right)}{\sum_{k=1}^{K} \exp \left(w_{k}^{T} x\right)} \sum_{i=1}^{K} \emptyset_{i}=1
$$

Note that when $\mathrm{K}=2$, the above becomes logistic functions:

$$
\begin{aligned}
& \emptyset_{0}\left(w_{0}^{T} x\right)=\frac{\exp \left(w_{0}^{T} x\right)}{\exp \left(w_{0}^{T} x\right)+\exp \left(w_{1}^{T} x\right)}=\frac{1}{1+\exp \left(-\left(w_{0}-w_{1}\right)^{T} x\right)}=\sigma\left(-w^{T} x\right) \\
& \emptyset_{1}\left(w_{1}^{T} x\right)=\frac{\exp \left(w_{1}^{T} x\right)}{\exp \left(w_{0}^{T} x\right)+\exp \left(w_{1}^{T} x\right)}=\frac{1}{1+\exp \left(\left(w_{0}-w_{1}\right)^{T} x\right)}=\sigma\left(-w^{T} x\right)
\end{aligned}
$$

Where $\mathrm{w}=\mathrm{w}_{0}-\mathrm{w}_{1}$

Now the hypothesis function is:

$h_{w}(x)=\left[\begin{array}{c}\emptyset_{1} \\ \vdots \\ \emptyset_{K}\end{array}\right]=\frac{1}{\sum_{k=1}^{K} \exp \left(w_{k}^{T} x\right)}\left[\begin{array}{c}\exp \left(w_{1}^{T} x\right) \\ \vdots \\ \exp \left(w_{K}^{T} x\right)\end{array}\right]$

The model parameters $\mathrm{w}_{1}, \ldots, \mathrm{w}_{\mathrm{K}}$ can be considered as the column vectors of the parameter array $\mathrm{W}=$ $\left[\mathrm{w}_{1}, \ldots, \mathrm{w}_{\mathrm{K}}\right]$. We note that these parameters are redundant as only $\mathrm{K}-1$ of the $\mathrm{K}$ components are independent, due to the constraint $\sum_{i=1}^{K} \varnothing_{i}=\mathbf{1}$.

Given the training data $\left.\boldsymbol{D}=\left\{\left(\boldsymbol{x}_{n}, \boldsymbol{y}_{n}\right), \boldsymbol{n}=\mathbf{1}, \ldots, \boldsymbol{N}\right\}\right\}$ 
, we get the likelihood function:

$$
L(W / D)=\prod_{n=1}^{N} p\left(y_{n} / x_{n}\right)=\prod_{n=1}^{N} \prod_{i=1}^{K} p\left(y_{n}=i / x_{n}\right)^{1\left\{y_{n}=i\right\}}
$$

Where we have defined:

$$
1\{y=i\}=\left\{\begin{array}{l}
1 \text { if } y=i \\
0 \text { if } y \neq i
\end{array}\right.
$$

The log likelihood is:

$$
\begin{gathered}
l(W / D)=\log L(w / D)=\sum_{n=1}^{N} \sum_{i=1}^{K} 1\left\{y_{n}=i\right\} \log p\left(y_{n}=i / x_{n}\right) \\
=\sum_{n=1}^{N} \sum_{i=1}^{K} 1\left\{y_{n}=i\right\} \log \left(\exp \left(w_{i}^{T} x_{n}\right) \mid \sum_{k=1}^{K} \exp \left(w_{k}^{T} x_{n}\right)\right) \\
=\sum_{n=1}^{N} \sum_{i=1}^{K} 1\left\{y_{n}=i\right\}\left(w_{i}^{T} x_{n}-\log \sum_{k=1}^{K} \exp \left(w_{k}^{T} x_{n}\right)\right)
\end{gathered}
$$

To find $\mathrm{w}_{\mathrm{j}}$ 's that maximize this log likelihood function, we use gradient ascend method based on the gradient

$$
\begin{gathered}
g_{l}\left(w_{j}\right)=\frac{d}{d w_{j}} l(W / D)=\frac{d}{d w_{j}} \sum_{n=1}^{N} \sum_{i=1}^{K} 1\left\{y_{n}=i\right\}\left(w_{i}^{T} x_{n}-\log \sum_{k=1}^{K} \exp \left(w_{k}^{T} x_{n}\right)\right) \\
=\sum_{n=1}^{N} 1\left\{y_{n}=j\right\}\left(x_{n}-\frac{\exp \left(w_{j}^{T} x_{n}\right)}{\sum_{k=1}^{K} \exp \left(w_{k}^{T} x_{n}\right)} x_{n}\right) \\
=\sum_{n=1}^{N} 1\left\{y_{n}=j\right\}\left(1-p\left(y_{n}=j / x_{n}\right)\right) x_{n}=\sum_{n=1}^{N} 1\left\{y_{n}=j\right\}\left(1-\emptyset_{i}\right) x_{n}
\end{gathered}
$$

Given the gradients $g_{1}\left(w_{j}\right)(k=1, \ldots, K)$, the gradient descent method can be used to find the $\mathrm{K}$ weight vectors

\begin{tabular}{|c|c|c|c|c|c|c|c|}
\hline \multicolumn{3}{|c|}{ Charging } & \multicolumn{2}{|c|}{ Discharging } & \multicolumn{3}{|c|}{ Operating Conditions } \\
\hline $\begin{array}{l}\text { Constant } \\
\text { Current } \\
\text { (A) }\end{array}$ & $\begin{array}{c}\text { Upper } \\
\text { voltage } \\
\text { limit (V) }\end{array}$ & $\begin{array}{c}\text { Cut - off } \\
\text { current } \\
(\mathrm{mA})\end{array}$ & $\begin{array}{l}\text { Constant } \\
\text { Current } \\
\text { (A) }\end{array}$ & $\begin{array}{l}\text { Cut-off } \\
\text { voltage } \\
\text { (V) }\end{array}$ & $\begin{array}{c}\text { Operating } \\
\text { temperature }\end{array}$ & $\begin{array}{c}\text { Initial } \\
\text { Capacity }\end{array}$ & $\begin{array}{c}\text { EOL } \\
\text { Capacity }\end{array}$ \\
\hline 1.5 & 4.2 & 20 & 2 & 2.7 & Room temp. & 1.86 & 1.3 \\
\hline
\end{tabular}
that minimize the cost function. Whether we should use softmax regression or $\mathrm{K}$ logistic regressions for a problem of $\mathrm{K}$ classes $\mathrm{C}_{1}, \ldots, \mathrm{C}_{\mathrm{K}}$ depends on the nature of the classes. The method of softmax regression is suitable if the $\mathrm{K}$ classes are mutually exclusive and independent, as assumed by the method. Else, $\mathrm{K}$ logistic regression binary classifiers are more reasonable.

\section{Experimental Results}

a. Battery Data Description And Visualization: Li-Ion battery dataset of NASA Prognostics Center of Excellence Data Repository is being employed, which consists of three distinct operating profiles of charging, discharging, and impedance at temperature. Constant current mode was used for charging purpose. A constant current of 1.5 Ampere was supplied till battery voltage become 4.2 Volts and later switched to constant voltage mode till charge current dropped to $20 \mathrm{~mA}$. Repeated charging \& discharging cycles result in early aging of the batteries. The experiment was terminated when the batteries reach end-of-life criteria, which was a 30\% fade-in rated capacity.

For the visualization purpose of the battery dataset, NumPy, Pandas, and Matplotlib libraries of Python language is utilized, and therefore the realization of appropriate programs for the visualization purpose was applied within the Jupyter Notebook Environment. Exploratory data visualization proved to represent quite an essential part within the development of an algorithm for battery RUL predictions because it helped in understanding the degradation nature of assorted battery parameters notably Voltage, Current and Discharging Capacity as a function of Cycle number in case of discharging cycles and Voltage and Current in case of charging cycles.

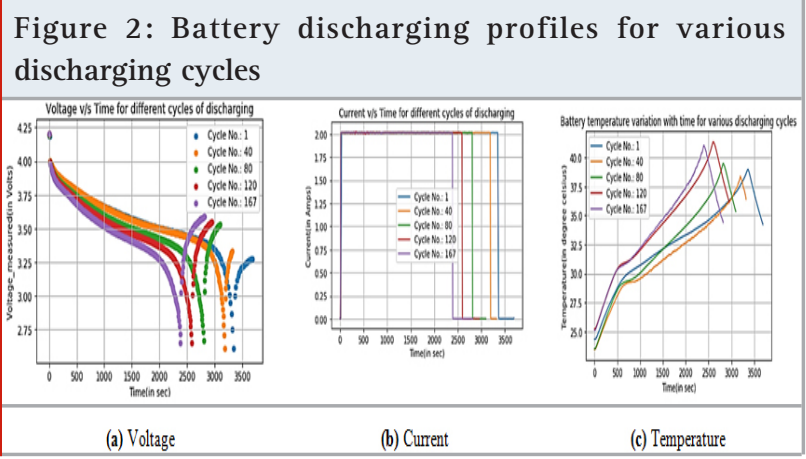

b. Remaining Useful Life (RUL) Prediction Of Battery Using Artificial Neural Network: The RUL prediction algorithm development problem was being approached as a multi-class classification problem, typically meaning that there are more than two distinct classes to be 
predicted. For example, there are a total of 167 distinct classes in the NASA Li-Ion Battery \#5 dataset. The key here for the development of the novel algorithm is the fields mentioned in Table \#2 classified as input labels and the output/target label consisting of a total of 167 classes numbered from 0-166. The apparent problem is efficiently implemented with the adequate help of a multi-layer neural network because the multi-class values typically require specialized handling.

Figure 3: Battery charging profiles for various charging cycles

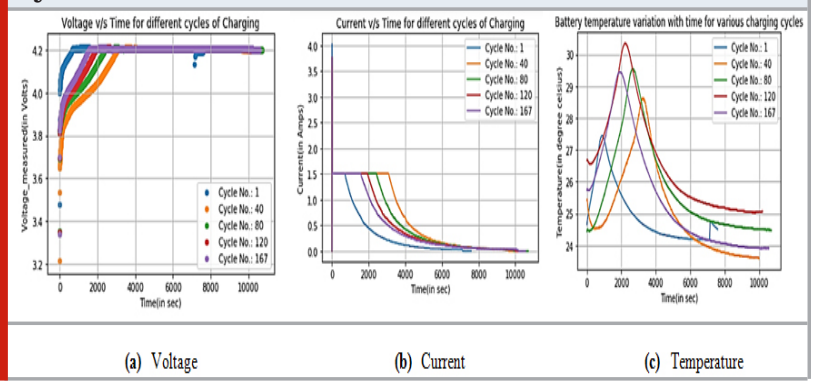

Figure 4: Capacity degradation over charging/discharging cycles

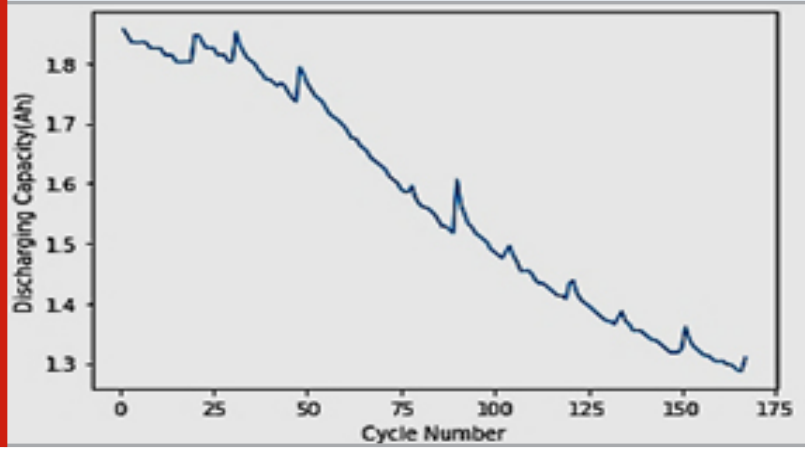

Figure 5: Overall workflow for prediction of Remaining Useful Life

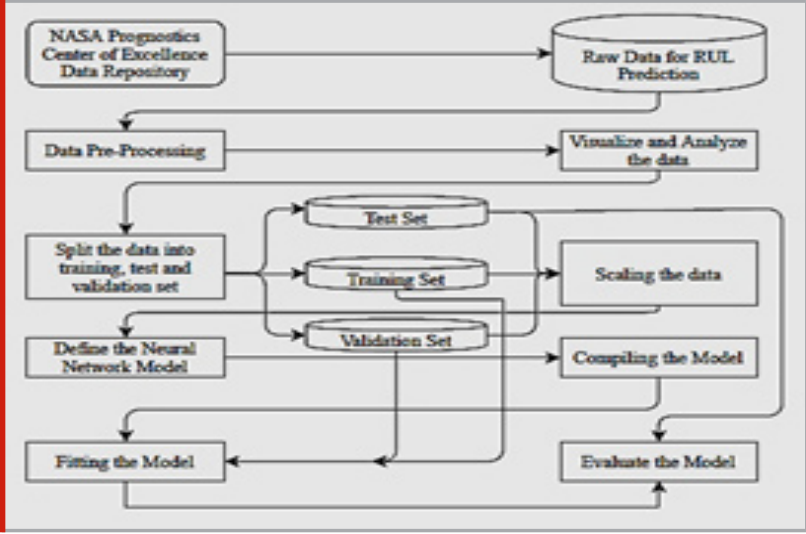

There is an identified total of 49,595 samples present in the dataset after carefully performing the datapreprocessing operations. These sample points can be further divided into training, validation, and test dataset.
The data fields available in the discharging cycle are represented respectively in Table \#2.

Table 2. Data fields containing the measurements

\begin{tabular}{|c|c|c|}
\hline & $\begin{array}{l}\text { Voltage_ } \\
\text { measured }\end{array}$ & $\begin{array}{c}\text { Battery terminal } \\
\text { voltage (Volts) }\end{array}$ \\
\hline \multirow[t]{5}{*}{$\begin{array}{l}\text { Input } \\
\text { Labels }\end{array}$} & Current_measured & $\begin{array}{l}\text { Battery output } \\
\text { current (Amps) }\end{array}$ \\
\hline & $\begin{array}{l}\text { Temperature_ } \\
\text { measured }\end{array}$ & $\begin{array}{c}\text { Battery temperature } \\
\text { (degree C) }\end{array}$ \\
\hline & Voltage_charge & $\begin{array}{l}\text { Voltage measured } \\
\text { at charger (Volts) }\end{array}$ \\
\hline & Time & $\begin{array}{l}\text { Time vector for } \\
\text { the cycle (sec) }\end{array}$ \\
\hline & $\begin{array}{l}\text { Discharge_ } \\
\text { Capacity }\end{array}$ & $\begin{array}{l}\text { Battery capacity for } \\
\text { discharge till } 2.7 \mathrm{~V} \text {. }\end{array}$ \\
\hline \multicolumn{3}{|c|}{$\begin{array}{l}\text { Charging Cycles left till battery reaches its End Of Life (EOL } \\
\text { criteria i.e., 30\% fade in its original capacity from 1.8Al } \\
\text { to } 1.3 \mathrm{Ah} \text {. }\end{array}$} \\
\hline
\end{tabular}

c. Preparing The Data And Designing The Model: The dataset can be loaded as a pandas data frame. Models in Keras are defined as a sequence of layers. Create a Sequential model and add layers one at a time until the desired network architecture is not achieved. Once the dataset is loaded, it can be categorized into a training set, validation set, and test set.

To understand the layers \& their types: Trial and error sometimes may lead to discovery of simple network structures. A network to be deployed should be adequate to capture the structure of matter. In this work, a fully connected network structure with three concealed layers is arranged. The layers are defined using the dense class. The numbers of nodes in the layer are specified as the first argument along with activation functions using activation argument. The Rectified linear unit activation function 'ReLU' is used on the primary three layers and the Softmax function was used within the output layer.

d. Compiling and Fitting The Model: In the system deployed, the backend itself selects an appropriate mechanism of network representation for training and supports predictions to operate on the system hardware. Training a network literally means identification of appropriate set of weights and mapping input-output of a dataset. The loss function accustomed to judging a bunch of weights must be considered. An optimizer is used to select appropriate weight for the network, considering optional metrics. The sparse categorical cross-entropy 
is utilized in this work due to the loss argument. The training procedure consists of adequate iterations on 'epochs' dataset that utilizes epochs argument. The total dataset rows considered prior to the model weights are updated within each epoch is termed as batch size that utilizes batch_size argument.

4. Evaluation of the Model: A neural network is trained on the training dataset and therefore the model is evaluated on the test dataset using the evaluate() function. The test dataset is passed as input and output i.e., X_test and y_test. This generates a prediction for every input and output pair and collects scores, including the average loss and metrics like accuracy. The evaluate() function returns a listing with dual values. The primary is going to be the loss of the model on the dataset and the secondary is going to be the correctness of the model on the utilized dataset. Figure \#4 depicts the loss and accuracy plot of the model implemented by us. Table \#4 indicates foremost classification metrics report. The metrics are expressed as a macro and weighted average. The metrics are calculated by using true and false positives \&t negatives for the anticipated classes. In order to ensure accuracy of the predictions, Precision, Recall \& F1 score are utilized.
Figure 6: Loss and Accuracy Plots

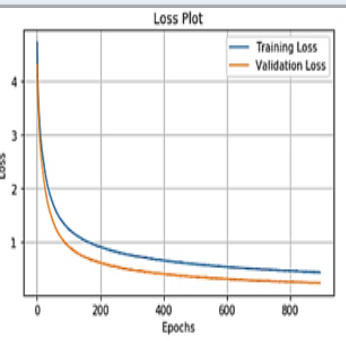

(a)

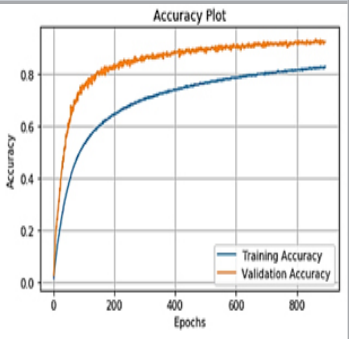

(b)
Figure 7: Comparison between true and predicted cycle numbers

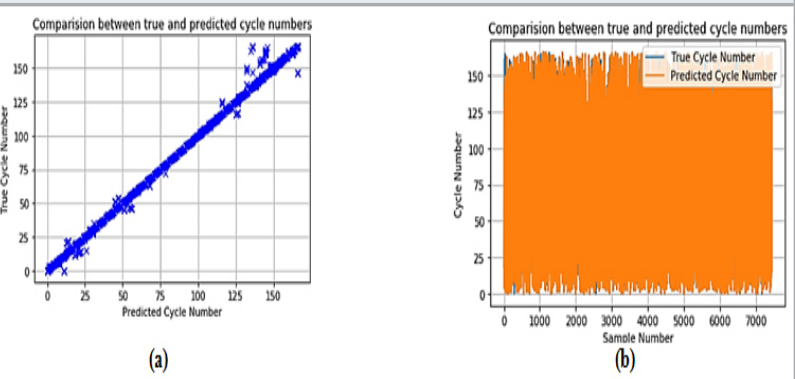

Table 3. Deviation between True and Predicted value for misclassified samples

\begin{tabular}{|l|c|c|c|c|c|}
\hline $\begin{array}{l}\text { True Value of } \\
\text { Remaining } \\
\text { Discharge Cycles }\end{array}$ & $\begin{array}{c}\text { Predicted Value } \\
\text { of Remaining } \\
\text { Discharge Cycles }\end{array}$ & $\begin{array}{c}\text { True } \\
\text { RUL (\%) }\end{array}$ & $\begin{array}{c}\text { Predicted } \\
\text { RUL (\%) }\end{array}$ & $\begin{array}{c}\text { Deviation from the } \\
\text { expected output } \\
\text { with sign }\end{array}$ & $\begin{array}{c}\text { Deviation from the } \\
\text { expected output } \\
\text { without sign }\end{array}$ \\
\hline 51 & 45 & 30.54 & 26.94 & 6 & 6 \\
\hline 139 & 140 & 83.23 & 83.83 & -1 & 1 \\
\hline 151 & 150 & 90.42 & 89.82 & 1 & 1 \\
\hline 149 & 148 & 89.22 & 89.22 & 1 & 1 \\
\hline 151 & 150 & 90.42 & 89.82 & 1 & 1 \\
\hline 19 & 20 & 11.38 & 11.97 & -1 & 21 \\
\hline 58 & 59 & 34.73 & 35.33 & -1 & 1 \\
\hline 155 & 134 & 92.81 & 80.23 & 21 & 1 \\
\hline 139 & 160 & 96.40 & 95.81 & 1 & 1 \\
\hline
\end{tabular}

Table 4. Classification Report

\begin{tabular}{|l|c|c|c|c|}
\hline & Precision & Recall & F1-Score & Support \\
\hline Accuracy & & & 0.94 & 7440 \\
\hline Macro average & 0.94 & 0.92 & 0.92 & 7440 \\
\hline Weighted average & 0.95 & 0.94 & 0.94 & 7440 \\
\hline
\end{tabular}

Precision: Precision is an ability of classifier to avoid false positives. It is a ratio of true positives to the combined true and false positives[8].
Precision $=\mathrm{TP} /(\mathrm{TP}+\mathrm{FP})$

Recall: How effectively a classifier can detect all positive occurrences in every class is termed as recall. It is the ratio of true positives to the aggregate of true positives and false negatives .

$$
\text { Recall }=\mathrm{TP} /(\mathrm{TP}+\mathrm{FN})
$$

F1 score: The F1 score is a weighted mean of precision and recall. A good F1 score is indicated by 1 while worst by 0 . Typically, F1 scores are estimated to be lesser than exactness measures due to use of accuracy and review. 
F1 Score $=2 *($ Recall $*$ Precision $) /($ Recall + Precision $)$

Table \#3 summarizes the True RUL (\%) and Predicted RUL (\%) and also the deviation between the true and predicted values for some of the random samples. And Figure \#7(a) approximates to linear nature whereas in Figure \#7(b) True Cycle Number curve almost overlaps with the Predicted Cycle Number curve. The abovementioned results justify the prediction efficacy of RUL by our model.

\section{CONCLUSION}

A novel RUL prediction model based on MultiLayer Neural Network which performs Multi-Class Classification by appending a softmax function to the last layer is illustrated. The reproduction result shows an optimum accuracy score of 93\% when six layers are used in the network. Experimental analysis indicates a decline in the accuracy when the number of layers deviates. The proposed technique does not rely on complicated battery electrochemical principles resulting in better versatility and can be deployed for RUL prediction of numerous kinds of batteries. According to the model metrics, the proposed method can be suitably deployed for RUL estimation of electric vehicle batteries. Moreover, by the virtue of cloud technology, a real-time RUL prediction about the battery and its maintenance timeline is possible. The RUL estimation tool proposed in this paper may benefit the upcoming automotive industry, particularly for electric vehicles.

\section{REFERENCES}

Algorithmia. (2019, December) algorithmia.com [Online]; https://algorithmia.com/blog/multiclass-classificationin-machine-learning Jianyu Liang, Yuanhao Shi, Jie Wen, Xiaoqiong Pang, Jianchao Zeng Jianfang Jia, "SOH and RUL Prediction of Lithium-Ion Batteries Based on Gaussian Process Regression with Indirect Health Indicators," Energies, vol. 13, no. 2, p. 375, Jan. 2020.

Open Calgary. (2018, June) nasa.gov [Online]; https:// data.nasa.gov/dataset/Li-ion-Battery-Aging-Datasets/ uj5r-zjdb Rahul Agarwal. (2019, Sep.) Medium [Online]; https://towardsdatascience.com/the-5-classificationevaluation-metrics-you-must-know-aa97784ff226 Ruye Wang. (2018, May) fourier.eng.hmc.edu [Online]; http:// fourier.eng.hmc.edu/e176/lectures/logisticRegression/ node2.html

Saloni Gupta. (2018, Jan.) GeeksforGeeks [Online]; https://www.geeksforgeeks.org/introduction-toartificial-neutral-networks/

Yohwan Choi, Won Jae Choi, Hee-Yeon Ryu, Hongseok Kim Kyungnam Park, "LSTM-based Battery Remaining Useful Life Prediction with Multi-Channel Charging Profiles," IEEE Access, vol. 8, pp. 20786-20798, 2020.

Zhiyong Zheng, Xiaoyong Zheng, Kunyuan Deng, Kai Gao, Heng Li, Bin Chen, Yingze Yang, Zhiwu Huang Jun Peng, ”A Data-Driven Method with Feature Enhancement and Adaptive Optimization for LithiumIon Battery Remaining Useful Life Prediction," Energies, vol. 13, no. 3, p. 752, Feb. 2020. 